\title{
Caracterização colorimétrica e da massa específica das madeiras comercializadas no Mato Grosso
}

\author{
Leonardo Antônio Moraes Zaque ${ }^{1 \star}$, Rafael Rodolfo de Melo $^{2}$, Edila Cristina de Souza ${ }^{1}$, Laercio Serenine \\ Junior $^{1}$ \\ ${ }^{1}$ Programa de Pós-Graduação em Ciências Florestais e Ambientais (PPGCFA), Universidade Federal de Mato Grosso \\ (UFMT), Mato Grosso, Brasil. \\ ${ }^{2}$ Universidade Federal do Semi-Árido (UFERSA), Mossoró, Rio Grande do Norte, Brasil.
}

\begin{abstract}
RESUMO O presente trabalho tem como objetivo caracterizar quantitativamente a cor e a massa específica da madeira das principais espécies comercializadas no estado de Mato Grosso. Foram selecionadas as trinta espécies mais comercializadas em Mato Grosso, segundo dados da Secretaria de Estado do Meio Ambiente. Foram determinados os parâmetros colorimétricos ( $L^{*}$, $\mathrm{a}^{*}, \mathrm{~b}^{*}, \mathrm{C}$ e h) a partir do sistema CIEL $L^{*} a^{*} b^{*}$, sendo realizada uma leitura no plano tangencial e outra no transversal, e também se determinou a massa específica aparente. Foram realizados o teste de Scott-Knott com posterior análise multivariada de Cluster e correlação de Pearson para o plano tangencial e massa específica, e realizou-se o teste de média para comparação dos parâmetros colorimétricos entre os planos tangencial e transversal. A partir da análise multivariada de Cluster, as madeiras foram separadas em três grupos. O primeiro grupo apresenta madeiras mais claras e com menores massas específicas, o segundo grupo as madeiras apresentaram dominância das colorações amarelo-amarronzado e marrom-claro e obtiveram valores intermediários para a massa específica em relação aos outros grupos, já o terceiro grupo as madeiras foram as mais escuras e obtiveram maiores massas específicas. A luminosidade, saturação e ângulo de tinta tiveram correlação com a massa específica.
\end{abstract}

Palavras-chave: propriedades organolépticas; cor; madeiras tropicais.

\section{Colorimetric characterization and density of wood marketed in Mato Grosso}

\begin{abstract}
This study aims to quantitatively characterize the color and mass specific of the wood of the main species marketed in the state of Mato Grosso. The thirty species most commercialized in Mato Grosso were selected, according to data from the State Secretary for the Environment. The colorimetric parameters $\left(L^{*}, a^{*}, b^{*}, C\right.$ and $h$ ) were determined from the CIEL $L^{*} a^{*} b^{*}$ system, with a reading in the tangential plane and another in the transverse plane, and the apparent density was determined. The Scott-Knott test was performed with a subsequent Multivariate Cluster and Pearson correlation analysis for the tangential plane and density, and the mean test was performed to compare the colorimetric parameters between the tangential and transverse planes. From the multivariate analysis of Cluster, the woods were separated into three groups. The first group presented lighter woods and with smaller densities, the second group the woods presented dominance of the yellow-brownish and light brown colorations and obtained intermediate values for the density in relation to the other groups, while the third group was the darkest and obtained the highest densities. The lightness, saturation and hue angle were correlated with density. Keywords: organoleptic properties; color; tropical woods.
\end{abstract}




\section{Introdução}

A colorimetria é uma técnica que, segundo Amorim et al. (2013), pode ser usada para a caracterização e qualificação da madeira. Embora a cor seja uma característica relevante tanto quanto às propriedades físicas, mecânicas, químicas e anatômicas, ela não tem sido suficientemente abordada (GARCIA et al., 2014).

A colorimetria é a medição quantitativa da cor para registrar uma cor objetivamente e traduzi-la em dados numéricos, sendo também interessante para estudar algumas características físicas, químicas e macroscópicas da madeira (LAVISCI et al., 1989). E para a medição da cor propriamente dita, são usados os colorímetros e os espectrofotocolorimetros (MORI et al., 2005). Conforme Rappold; Smith (2004), o colorímetro possui uma série de filtros e fotodetectores para quantificar a cor dos materiais expostos, já o espectrofotômetro fornece uma análise da intensidade da luz em vários comprimentos de onda da amostra em termos de reflexão ou transmissão ou espectral (LEÃO, et al., 2005). Um dos sistemas mais usados para a medição de cores é o sistema $\operatorname{CIE} L^{\star} a^{\star} b^{\star}$, que se fundamenta em três elementos: a luminosidade, a tonalidade, e a saturação (BARROS et al., 2014).

De acordo com Camargos; Gonçalez (2001), a cor é uma das propriedades mais essenciais para a identificação e indicação de usos de madeiras, especialmente quando relacionada aos aspectos de textura e desenho. Estes autores ainda ressaltam que a cor pode ser modificada por diversos fatores, como alterações no teor de umidade, na temperatura, por deteriorações causadas pelo ataque de organismos xilófagos ou por reações fotoquímicas dos elementos presentes na sua estrutura.

As propriedades colorimétricas de algumas madeiras tropicais são almejadas para fabricação de móveis, porém a exploração inadequada de florestas tropicais e o maior rigor dos órgãos de fiscalização estão contribuindo para a aumento do preço destes materiais ou até extinguindo-os no mercado, ocasionando um desequilíbrio entre oferta e procura (ZANUNCIO et al., 2014).

A cor da madeira varia amplamente entre espécies, sendo um fator importante para estabelecer a qualidade e uso da mesma. É difícil descrever a aparência da madeira usando valores físicos, porque a superfície da madeira nunca é expressa com uma única cor. Desse modo, a colorimetria é um método para obter informações exatas sobre a cor de uma madeira (NISHINO et al., 1998).

O presente trabalho tem como objetivo caracterizar quantitativamente a cor e a massa específica da madeira das principais espécies comercializadas no estado de Mato Grosso.

\section{Material e Métodos}

\section{Seleção das espécies}

A seleção das espécies foi realizada com base no relatório parcial de Beneficiamento e Comércio de Produtos da Madeira por Espécie Florestal, disponibilizados pela Secretária de Estado do Meio Ambiente de Mato Grosso (SEMA-MT, 2016). Foram selecionadas trinta espécies diferentes entre as mais comercializadas no estado de Mato Grosso, das quais foram obtidas amostras para a caracterização colorimétrica.

As espécies selecionadas mais comercializadas em ordem de importância foram Erisma uncinatum Warm (Cedrinho), Goupia glabra Aubl. (Cupiúba), Qualea sp. (Cambará), Manilkara sp. (Maçaranduba), Apuleia sp. (Garapeira), Hymenolobium sp. (Angelim), Dipteryx sp. (Champanhe), Trattinnickia sp. (Amescla), Mezilaurus itauba (Meissn.) (Itaúba), Tabebuia sp. (Ipê), Hymenaea sp. (Jatobá), Protium sp. (Breu), Clarisia racemosa Ruiz e Pavon (Amarelinho), Cedrelinga catenaeformis Ducke (Cedrão), Simarouba amara 
Aubl. (Caixeta), Carinana sp. (Jequitibá), Ocotea sp. (Canela), Bagassa guianensis Aubl. (Tatajuba), Enterolobium contortisiliquum (Vell.) Morong. (Orelha-de-macaco), Astronium sp. (Muiracatiara), Castilla ulei Warb. (Caúcho), Enterolobium schomburgkii (Benth.) Benth. (Faveira-dura), Parkia sp. (Faveira), Couratari sp. (Tauri), Sclerolobium sp. (Tachí), Schizolobium amazonicum (Huber) Ducke. (Pinhocuiabano), Pouteria sp. (Abiurana), Guarea sp. (Marinheiro), Dialium guianense (Aubl.) Sandwith (Jutaí) e Vochysia sp (Cambará-rosa).

\section{Coleta das amostras}

As amostras foram coletadas em serraria de diferentes cidades da Região Norte de Mato Grosso, principal polo madeireiro do estado, sendo 5 amostras por espécie de tábuas diferentes. Estas foram encaminhadas para o Instituto de Defesa Agropecuária do Estado de Mato Grosso (INDEA$\mathrm{MT}$ ), onde foram comparadas com as amostras-padrão da Xiloteca do Laboratório de Tecnologia da Madeira. Com base nessa comparação e, com o auxílio da análise de um especialista do Laboratório, foram feitas as identificações das espécies. Quando não foi possível identificar a nível de espécie, identificou-se a nível de gênero.

Para cada espécie foram confeccionadas cinco amostras de 5,0 x 1,0 x 12,0 cm nos sentidos tangencial, radial e axial, respectivamente, e lixadas com lixa de granulometria de 200, para que a oxidação das camadas superiores da madeira não afetasse a sua tonalidade natural. Essas amostras foram utilizadas para a determinação da massa específica e dos parâmetros colorimétricos.

\section{Determinação da massa específica aparente}

A massa específica aparente foi obtida pela relação da massa e volume (Equação 1), sendo realizada 5 repetições por espécie. A massa foi aferida através da pesagem em balança analítica de precisão $(0,01 \mathrm{~g})$ e o volume estabelecido pela mensuração das direções axial, longitudinal radial e longitudinal tangencial de cada amostra, usando um paquímetro $(0,01 \mathrm{~mm})$.

$$
\rho=\mathrm{m} / \mathrm{v} \quad \text { (Equação 1) }
$$

Em que: $\rho=$ massa específica, $\mathrm{em} \mathrm{g} / \mathrm{cm}^{3} ; \mathrm{m}=$ massa, $\mathrm{em}$ gramas; $\mathrm{v}=$ volume, $\mathrm{em}^{3}$.

As massas específicas das madeiras (Tabela 1) foram classificadas de acordo com a classificação proposta por INDEA (2011).

Tabela 1. Classificação da massa específica da madeira.

Table 1. Classification of the wood density.

\begin{tabular}{cc}
\hline Massa específica & Classificação \\
\hline 0,00 a $0,40 \mathrm{~g} / \mathrm{cm}^{3}$ & Muito leve \\
0,41 a $0,55 \mathrm{~g} / \mathrm{cm}^{3}$ & Leve \\
0,56 a $0,75 \mathrm{~g} / \mathrm{cm}^{3}$ & Moderadamente pesada \\
0,76 a $0,95 \mathrm{~g} / \mathrm{cm}^{3}$ & Pesada \\
$0,96 \mathrm{~g} / \mathrm{cm}^{3}$ acima & Muito pesada
\end{tabular}

\section{Caracterização colorimétrica}

A caracterização colorimétrica foi realizada por meio de um espectrofotocolorímetro com resolução de $3 \mathrm{~nm}$ de iluminação difusa, usando iluminante D65 composto por lâmpada de xenônio com ângulo de observação $10^{\circ} \mathrm{e}$ área de iluminação $11 \mathrm{~mm}$ de diâmetro. Foram feitas duas leituras em cada uma das 5 amostras das espécies, uma no plano tangencial e a outra no plano transversal, para obter os parâmetros colorimétricos a partir do sistema $\operatorname{CIE} L^{*} a^{*} b^{*}$ de 1976, conforme recomendado pela Comission International de L'Eclairage (CIE).

Os parâmetros avaliados foram: $L^{\star}$ (luminosidade ou claridade), que varia entre 0 e 100, em que zero caracteriza o preto e cem o branco, também denominado eixo cinza; as 
coordenadas cromáticas $\mathrm{a}^{\star} \mathrm{e}^{\star}$, que representam as posições dos pontos de cores sobre os eixos verde-vermelho e azulamarelo, respectivamente e assume valores de 0 e 60 , sendo que o número positivo indica o vermelho e amarelo, e os números negativos o verde e azul; a saturação de cor (C) corresponde a distância do eixo de luminosidade, e quanto maior a distância do eixo cinzento, mais saturada será a cor; e o ângulo de tonalidade no círculo (h), que representa a dominância de um componente de tonalidade em alguma cor, sendo que esses dois últimos parâmetros são calculadas a partir das coordenadas cromáticas $\mathrm{a}^{\star}$ e $\mathrm{b}^{*}$ (PINCELLI et al., 2012)

Para auxiliar na caracterização do padrão de cor das amostras, foi usada a tabela de cores com variação numéricas das variáveis proposta por Camargo; Gonçalez (2001). Foram atribuídas novas denominações de cor de acordo com os parâmetros colorimétricos observados para as cores das madeiras que não se enquadraram nas propostas destes autores.

\section{Análise dos dados}

As comparações da massa específica, dos parâmetros colorimétricos e entre os planos tangencial e transversal foram avaliadas por meio de análise de variância (ANOVA) com posterior comparação pelo teste de Scott-Knott a um nível de 5\%, para os parâmetros detectados como significativos pelo teste de $\mathrm{F}$.

Para a cor e massa específica no plano tangencial foi realizada análise de agrupamento de Cluster, pelo método de Ward, usando a distância euclidiana padronizada e a linha de corte foi definida com base na variabilidade das características macroscópicas das madeiras avaliadas. Foi feita a correlação simples de Pearson entre a massa específica e os parâmetros cromáticos do plano tangencial.

\section{Resultados e Discussão}

\section{Massa específica}

Na Tabela 2, são apresentadas as massas específicas das madeiras estudadas. As madeiras classificadas como moderadamente pesadas foram as que tiveram maior representatividade com 36,6\%, seguidas pelas madeiras pesadas e muito pesadas que representaram $26,66 \%$ e $20 \%$, respectivamente, já as madeiras consideradas leves e muito leves corresponderam a $13,3 \%$ e $3,33 \%$, respectivamente.

De acordo com Dias Junior et al. (2014), uma madeira mais pesada apresenta difícil trabalhabilidade, enquanto que uma madeira leve é mais fácil de ser trabalhada e mais macia. Portanto, as madeiras com alta massa específica são recomendadas para a construção civil pesada externa e interna, já as madeiras com massa específica de média a alta para assoalhos domésticos e industriais. Na construção civil leve externa e interna, é recomendado o uso de madeiras de baixa a média massa específica (GROBÉRIO; LAHR, 2002).

Os resultados da massa específica verificados para as madeiras de Mezilaurus itauba $\left(0,89 \mathrm{~g} / \mathrm{cm}^{3}\right)$, Simarouba amara $\left(0,41 \mathrm{~g} / \mathrm{cm}^{3}\right)$ e Schizolobium amazonicum $\left(0,34 \mathrm{~g} / \mathrm{cm}^{3}\right)$ corroboram com Romanini et al. (2014), Stangerlin et al. (2013) e Silva et al. (2013) que destacaram valores de 0,80 $\mathrm{g} / \mathrm{cm}^{3}, 0,40 \mathrm{~g} / \mathrm{cm}^{3}$ e $0,32 \mathrm{~g} / \mathrm{cm}^{3}$ para cada uma das espécies respectivamente.

Dias Júnior et al. (2014), estudando a espécie Tabebuia alba, encontraram densidade de $1,18 \mathrm{~g} / \mathrm{cm}^{3}$, valor semelhante ao deste trabalho, e recomendaram que seja usada pela indústria moveleira na fabricação de móveis ou de seus componentes. 
Tabela 2. Massa específica das espécies comercializadas no estado de Mato Grosso no ano de 2015.

Table 2. Density of the species traded in the state of Mato Grosso in the year 2015.

\begin{tabular}{|c|c|c|}
\hline Espécies & Massa Específica Média $\left(\mathrm{g} / \mathrm{cm}^{3}\right) / \mathrm{CV}(\%)$ & Classificação $^{1}$ \\
\hline Schizolobium amazonicum & $0,38 \mathrm{G}(12,76)$ & Muito leve \\
\hline Simarouba amara & $0,41 \mathrm{G}(3,18)$ & Leve \\
\hline Enterolobium contortisiliquum & $0,46 \mathrm{G}(9,55)$ & Leve \\
\hline Castilla ulei & $0,44 \mathrm{G}(14,55)$ & Leve \\
\hline Vochysia sp & $0,55 \mathrm{~F}(11,54)$ & Leve \\
\hline Parkia sp & $0,56 \mathrm{~F}(4,45)$ & Moderadamente pesada \\
\hline Couratarisp & $0,58 \mathrm{~F}(7,61)$ & Moderadamente pesada \\
\hline Trattinnickia sp & $0,58 \mathrm{~F}(3,32)$ & Moderadamente pesada \\
\hline Sclerolobium sp & $0,60 \mathrm{~F}(1,90)$ & Moderadamente pesada \\
\hline Cariniana sp & $0,60 \mathrm{~F}(1,49)$ & Moderadamente pesada \\
\hline Cedrelinga catenaeformis & $0,62 \mathrm{~F}(13,33)$ & Moderadamente pesada \\
\hline Erisma uncinatum & $0,63 \mathrm{~F}(5,31)$ & Moderadamente pesada \\
\hline Guarea sp & $0,68 \mathrm{E}(12,24)$ & Moderadamente pesada \\
\hline Qualea sp & $0,73 \mathrm{D}(5,86)$ & Moderadamente pesada \\
\hline Protium sp & $0,74 \mathrm{D}(4,21)$ & Moderadamente pesada \\
\hline Ocotea sp & $0,73 \mathrm{D}(2,67)$ & Moderadamente pesada \\
\hline Hymenolobium sp & $0,80 \mathrm{D}(9,62)$ & Pesada \\
\hline Clarisia racemosa & $0,82 \mathrm{C}(8,29)$ & Pesada \\
\hline Bagassa guianensis & $0,84 \mathrm{C}(3,95)$ & Pesada \\
\hline Pouteria sp & $0,86 \mathrm{C}(1,33)$ & Pesada \\
\hline Apuleia sp & $0,86 \mathrm{C}(2,96)$ & Pesada \\
\hline Astronium sp & $0,87 \mathrm{C}(13,64)$ & Pesada \\
\hline Goupia glabra & $0,88 \mathrm{C}(6,45)$ & Pesada \\
\hline Enterolobium schomburgkii & $0,89 \mathrm{~B}(18,08)$ & Pesada \\
\hline Mezilaurus itauba & $0,89 \mathrm{C}(5,54)$ & Pesada \\
\hline Hymenaea sp & 0,97 B $(6,38)$ & Muito pesada \\
\hline Dialium guianense & 0,99 B $(1,83)$ & Muito pesada \\
\hline Manilkara sp & 1,01 B $(0,89)$ & Muito pesada \\
\hline Dipteryx sp & 1,06 A $(1,40)$ & Muito pesada \\
\hline Tabebuia sp & 1,09 A $(8,67)$ & Muito pesada \\
\hline
\end{tabular}

Médias seguidas de mesma letra em cada coluna, não diferem estatisticamente pelo teste de Scott-Knott em 5\% de significância de probabilidade; CV: coeficiente de variação; ${ }^{1}$ Classificação de INDEA (2011).

\section{Características colorimétricas}

Foram observadas variações consideráveis para todos os parâmetros colorimétricos avaliados (Tabela 3). Para a luminosidade $\left(\mathrm{L}^{*}\right)$, os valores variaram de 40,44 a 82,46. Nishino et al. (1998), que estudaram noventa e sete espécies da Guiana Francesa, sendo que dessas espécies dezesseis são as mesmas do presente trabalho. Esses autores encontraram valores entre 36 e 78,98, os quais são semelhantes ao deste trabalho, ou seja, ocorreu grande variação na luminosidade. Na Tabela 4 são apresentadas as cores do plano tangencial conforme Camargo; Gonçalez (2001). 
Tabela 3. Parâmetros colorimétricos do plano tangencial de espécies comercializadas no estado de Mato Grosso no ano de 2015.

Table 3. Colorimetric parameters acquired in the tangential direction of the wood commercialized in the state of Mato Grosso in the year 2015.

\begin{tabular}{|c|c|c|c|c|c|}
\hline Espécies & $\mathbf{L}^{*}$ & $\mathbf{a}^{*}$ & $\mathbf{b}^{*}$ & $\mathrm{C}$ & h \\
\hline Schizolobium amazonicum & $81,10 \mathrm{~A}$ & $5,77 \mathrm{E}$ & $26,38 \mathrm{C}$ & $27,06 \mathrm{~B}$ & $77,47 \mathrm{~B}$ \\
\hline Simarouba amara & $82,46 \mathrm{~A}$ & $3,81 \mathrm{H}$ & $24,07 \mathrm{C}$ & $24,38 \mathrm{C}$ & $81,03 \mathrm{~A}$ \\
\hline Parkia sp & $75,16 \mathrm{~B}$ & $6,97 \mathrm{~F}$ & $28,90 \mathrm{~B}$ & $29,76 \mathrm{~B}$ & $76,23 \mathrm{~B}$ \\
\hline Couratari sp & $69,07 \mathrm{C}$ & $9,08 \mathrm{E}$ & $26,80 \mathrm{C}$ & $28,33 \mathrm{~B}$ & $71,40 \mathrm{C}$ \\
\hline Castilla ulei & $70,12 \mathrm{C}$ & $7,48 \mathrm{~F}$ & $27,73 B$ & $28,72 B$ & $74,90 \mathrm{~B}$ \\
\hline Enterolobium schomburgkii & $57,50 \mathrm{E}$ & $12,01 \mathrm{D}$ & $27,24 \mathrm{~A}$ & $29,8 \mathrm{~B}$ & $66,20 \mathrm{D}$ \\
\hline Bagassa guianensis & $54,21 \mathrm{~F}$ & $11,68 \mathrm{D}$ & $26,62 B$ & $29,09 \mathrm{~B}$ & $66,22 \mathrm{D}$ \\
\hline Clarisia racemosa & $57,08 \mathrm{E}$ & $10,65 \mathrm{D}$ & $30,63 \mathrm{~A}$ & $32,46 \mathrm{~A}$ & $70,73 \mathrm{C}$ \\
\hline Apuleia sp & $61,44 \mathrm{D}$ & $13,13 \mathrm{C}$ & $30,59 \mathrm{~A}$ & $33,34 \mathrm{~A}$ & $66,71 \mathrm{D}$ \\
\hline Sclerolobium $\mathrm{sp}$ & $61,92 \mathrm{D}$ & $10,4 \mathrm{D}$ & $21,45 \mathrm{D}$ & $23,84 \mathrm{C}$ & $64,14 \mathrm{E}$ \\
\hline Vochysia sp & $63,05 \mathrm{D}$ & $14,3 \mathrm{~B}$ & $22,70 \mathrm{D}$ & $26,88 \mathrm{~B}$ & $57,61 \mathrm{~F}$ \\
\hline Hymenolobium sp & $61,28 \mathrm{D}$ & $13,09 \mathrm{C}$ & $24,73 \mathrm{C}$ & $27,99 \mathrm{~B}$ & $62,07 \mathrm{E}$ \\
\hline Trattinnickia sp & $66,29 \mathrm{C}$ & $11,4 \mathrm{D}$ & $26,9 \mathrm{C}$ & $29,34 \mathrm{~B}$ & $67,48 \mathrm{D}$ \\
\hline Pouteria sp & $51,25 \mathrm{~F}$ & $15,45 \mathrm{~B}$ & $22,83 \mathrm{D}$ & $27,58 \mathrm{~B}$ & $55,83 \mathrm{G}$ \\
\hline Astronium sp & $46,87 \mathrm{G}$ & $15,75 \mathrm{~B}$ & $23,93 \mathrm{C}$ & $28,65 \mathrm{~B}$ & $56,62 \mathrm{G}$ \\
\hline Hymenaea sp & $49,27 \mathrm{G}$ & $17,96 \mathrm{~A}$ & $25,63 \mathrm{C}$ & $31,36 \mathrm{~A}$ & $54,64 \mathrm{G}$ \\
\hline Guarea sp & $60,27 \mathrm{D}$ & $16,20 \mathrm{~B}$ & $26,33 \mathrm{C}$ & $30,93 \mathrm{~A}$ & $58,38 \mathrm{~F}$ \\
\hline Protium sp & $57,42 \mathrm{E}$ & $14,37 \mathrm{~B}$ & $27,84 \mathrm{~B}$ & $31,34 \mathrm{~A}$ & $62,69 \mathrm{E}$ \\
\hline Qualea sp & $52,61 \mathrm{~F}$ & $15,39 \mathrm{~B}$ & $25,78 \mathrm{C}$ & $30,03 \mathrm{~B}$ & $59,17 \mathrm{~F}$ \\
\hline Goupia glabra & $54,47 \mathrm{~F}$ & $15,39 \mathrm{~B}$ & $25,55 \mathrm{C}$ & $29,91 B$ & $58,72 \mathrm{~F}$ \\
\hline Cedrelinga catenaeformis & $53,19 \mathrm{~F}$ & $11,31 \mathrm{D}$ & $19,34 \mathrm{E}$ & $22,41 \mathrm{D}$ & $59,72 \mathrm{~F}$ \\
\hline Enterolobium contortisiliquum & $54,81 \mathrm{~F}$ & $11,97 \mathrm{D}$ & $21,26 \mathrm{D}$ & $24,41 \mathrm{C}$ & $60,59 \mathrm{~F}$ \\
\hline Dipteryx sp & $53,74 \mathrm{~F}$ & $12,34 \mathrm{C}$ & $22,27 \mathrm{D}$ & $25,48 \mathrm{C}$ & $60,95 \mathrm{~F}$ \\
\hline Tabebuia sp & $47,42 \mathrm{G}$ & $6,97 \mathrm{~F}$ & $19,26 \mathrm{E}$ & $20,51 \mathrm{D}$ & $70,16 \mathrm{C}$ \\
\hline Mezilaurus itauba & $48,18 \mathrm{G}$ & $5,41 \mathrm{G}$ & $21,19 \mathrm{D}$ & $21,87 \mathrm{D}$ & $75,67 \mathrm{~B}$ \\
\hline Dialium guianense & $40,43 \mathrm{H}$ & $13,33 \mathrm{C}$ & $17,14 \mathrm{~F}$ & $21,73 \mathrm{D}$ & $51,91 \mathrm{H}$ \\
\hline Manilkara sp & $41,68 \mathrm{H}$ & $12,97 \mathrm{C}$ & $14,78 \mathrm{~F}$ & $19,68 \mathrm{D}$ & $48,87 \mathrm{H}$ \\
\hline Ocotea sp & $41,41 \mathrm{H}$ & $10,73 \mathrm{D}$ & $18,85 \mathrm{E}$ & $21,69 \mathrm{D}$ & $60,31 \mathrm{~F}$ \\
\hline Erisma uncinatum & $47,31 \mathrm{G}$ & $12,68 \mathrm{C}$ & $18,76 \mathrm{E}$ & $22,66 \mathrm{D}$ & $56,00 \mathrm{G}$ \\
\hline Cariniana sp & $50,12 \mathrm{~F}$ & $13,68 \mathrm{C}$ & $19,71 \mathrm{E}$ & $24,00 \mathrm{C}$ & $55,18 \mathrm{G}$ \\
\hline
\end{tabular}

As espécies Schizolobium amazonicum, Simarouba amara

e Parkia sp foram as espécies mais claras, pois apresentaram maiores valores de luminosidade $\left(L^{\star}\right)$ e valores do ângulo de tinta (h) mais próximos da coordenada cromática do eixo amarelo-azul $\left(\mathrm{b}^{\star}\right)$. Já as espécies mais escuras foram Dialium guianense, Manilkara sp. e Ocotea sp., pois obtiveram os menores valores para luminosidade e valores altos para a coordenada cromática do eixo verde-vermelho $\left(a^{\star}\right)$. Estes resultados corroboram com o de Silva et al. (2017), que averiguaram como a espécie mais clara a Simarouba amara e a mais escura a Dialium guianense.
Todas as espécies apresentaram a superfície tangencial mais amarelada do que avermelhada, principalmente a Clarisia racemosa, Apuleia sp. e Parkia sp. que apresentaram maiores valores para o eixo $b^{*}$, este parâmetro teve mais influência da saturação (C) do que o parâmetro do eixo verdevermelho, sendo estas espécies as mais puras. Silva et al. (2017), encontraram maiores valores de $b^{\star}$ nas espécies de Morus sp., Clarisia racemosa e Apuleia leiocarpa sendo consideradas as mais saturadas. Grey (2006), explica que uma cor mais saturada será mais pura e vibrante, enquanto uma 
cor menos saturada aparecerá mais fraca e com menor pureza.

Tabela 4. Cores do plano tangencial das espécies comercializadas do estado de Mato Grosso no ano de 2015.

Table 4. Colors based on the tangential direction of the wood commercialized in the state of Mato Grosso in the year 2015.

\begin{tabular}{|c|c|}
\hline Espécies & Cores \\
\hline Schizolobium amazonicum & Esbranquiçado $^{\star *}$ \\
\hline Simarouba amara & Branco-acinzentado* \\
\hline Parkia sp & Esbranquiçado ${ }^{* *}$ \\
\hline Couratari sp & Amarelo-claro* \\
\hline Castilla ulei & Amarelo-claro* \\
\hline Enterolobium schomburgkii & Marrom-oliva* \\
\hline Bagassa guianensis & Marrom-oliva* \\
\hline Clarisia racemosa & Amarelo-acastanhado ${ }^{\star *}$ \\
\hline Apuleia sp & Bege-amarelado ${ }^{* *}$ \\
\hline Sclerolobium sp & Rosa-acinzentado* \\
\hline Vochysia sp & $\operatorname{Rosa}^{*}$ \\
\hline Hymenolobium sp & $\operatorname{Rosa}^{*}$ \\
\hline Trattinnickia sp & Marrom-oliva* \\
\hline Pouteria sp & $\operatorname{Rosa}^{*}$ \\
\hline Astronium sp & Amarelo-amarronzado* \\
\hline Hymenaea sp & Amarelo-amarronzado* \\
\hline Guarea sp & Amarelo-amarronzado* \\
\hline Protium sp & Amarelo-amarronzado ${ }^{*}$ \\
\hline Qualea sp & Amarelo-amarronzado* \\
\hline Goupia glabra & Amarelo-amarronzado* \\
\hline Cedrelinga catenaeformis & Marrom-claro* $^{*}$ \\
\hline $\begin{array}{c}\text { Enterolobium } \\
\text { contortisiliquum }\end{array}$ & Marrom-claro* $^{*}$ \\
\hline Dipteryx sp & Marrom claro* \\
\hline Tabebuia sp & Oliva $^{*}$ \\
\hline Mezilaurus itauba & Castanho-oliváceo $^{\star *}$ \\
\hline Dialium guianense & Marrom-escuro* \\
\hline Manilkara sp & Marrom escuro* \\
\hline Ocotea sp & Pardo-amarelado ${ }^{\star *}$ \\
\hline Erisma uncinatum & Marrom-claro $^{\star}$ \\
\hline Cariniana sp & Marrom-avermelhado* \\
\hline
\end{tabular}

${ }^{*}$ Cores determinadas a partir da tabela proposta por Camargo e Gonçalez (2001); ${ }^{\star *}$ Cores nomeadas pelos autores.

As madeiras de Dialium guianense, Couratari sp., Dipteryx sp., Qualea sp., apresentaram para os parâmetros colorimétricos valores médios semelhantes aos encontrados por Silva et al. (2017), Costa et al. (2011), Paula et al. (2016), Nishino et al. (1998). Porém para as espécies Hymenaea sp., Cariniana sp., e Parkia sp., os valores encontrados foram diferentes dos encontrados por Costa et al. (2011), Barros et al. (2014) e Nishino et al. (1998), provavelmente essa diferença ocorreu por serem de origens diferentes.

A partir da análise multivariada de Cluster, as madeiras das espécies florestais avaliadas foram separadas em três grupos (Figura 1), na qual foram avaliados os pontos de alta mudança de nível (CRUZ; REGAZZI, 2001; MANLY, 2008).

Figura 1. Agrupamento de cluster da cor e massa específica da madeira do plano tangencial das principais espécies florestais comercializadas no Mato Grosso.

Figure 1. Clustering of color and density based on the tangential direction of the main wood species commercialized in Mato Grosso.

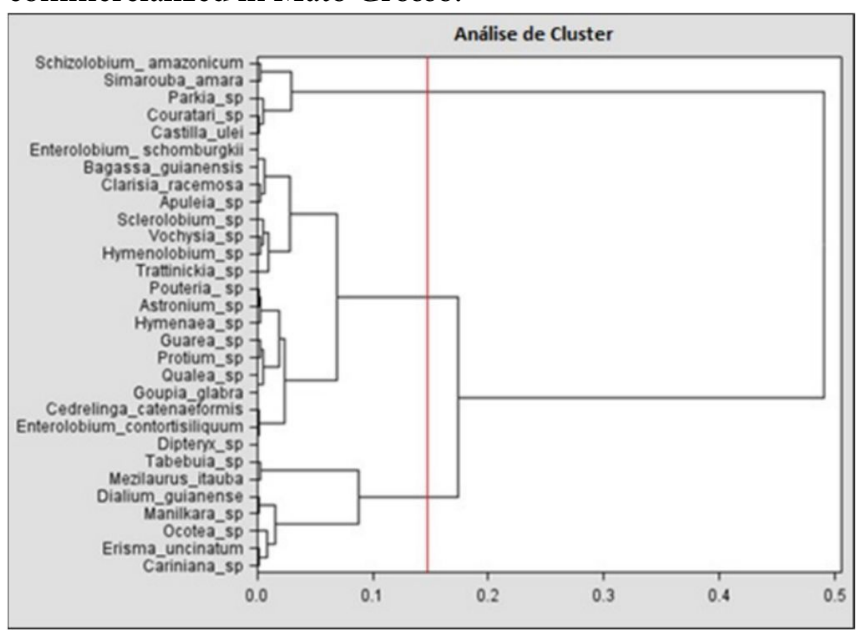

O grupo 1 foi composto pelas espécies de cores mais claras e com baixos valores de massa específica como o Schizolobium amazonicum, Simarouba amara, Parkia sp., Couratari sp. e Castilla ulei e representaram 16,7\% das espécies. Essas espécies apresentaram valores altos para luminosidade e ângulo de tinta em comparação com os outros grupos, porém tiveram baixos valores para o eixo verdevermelho $\left(a^{\star}\right)$.

Melo et al. (2013) explicaram que baixos valores para o eixo verde-vermelho $\left(\mathrm{a}^{\star}\right)$ podem indicar um baixo percentual de extrativos presentes na madeira. Pertuzzatti et al. (2016), estudando a espécie Pinus elliotti var. elliotti, encontraram valores colorimétricos semelhantes ao deste grupo, indicando 
que essas espécies por serem claras podem ser possíveis substitutas de uma das mais madeiras comercializadas no Brasil. Conforme Camargos; Gonçalez (2001), a madeira é considerada de cor clara quando o valor da luminosidade for acima de 56. Como todas as espécies deste grupo obtiveram valores de luminosidade acima deste valor, permaneceram juntas.

O grupo 2 foi composto pelas espécies Enterolobium schomburgkii, Bagassa guianensis, Clarisia racemosa, Apuleia sp., Sclerolobium sp., Vochysia sp., Hymenolobium sp., Trattinnickia sp., Pouteria sp., Astronium sp., Hymenaea sp., Guarea sp., Protium sp., Qualea sp., Goupia glabra, Cedrelinga catenaeformis, Enterolobium contortisiliquum e Dipteryx sp. representaram 60\% das espécies. Essas espécies apresentaram dominância das colorações amareloamarronzado e marrom-claro, e obtiveram valores intermediários para a massa específica e parâmetros colorimétricos em relação aos grupos 1 e 3 .

Esse grupo apresentou os maiores valores para o eixo verde-vermelho e para a saturação (C) em comparação com outros grupos. Já para os parâmetros de luminosidade e ângulo de tinta, apresentou valores inferiores ao do grupo $1 \mathrm{e}$ superiores ao grupo 3. As espécies Bagassa guianensis, Qualea sp., Clarisia racemosa, Dipteryx odorata, Hymenolobium petraeum e Apuleia leiocarpa também foram agrupadas em um mesmo grupo nos estudos realizados Silva et al. (2017).

O grupo 3 compreendeu $23,3 \%$ das espécies, sendo integrado por Tabebuia sp, Mezilaurus itauba, Dialium guianense, Manilkara sp., Ocotea sp, Erisma uncinatum e Cariniana sp e apresentaram cores mais escuras com predominância do marrom-escuro e foram as que apresentaram maiores valores para massa específica. Este grupo apresentou valores superiores do eixo verde-vermelho em relação ao grupo 1, e obteve os menores valores para o eixo azul-amarelo, luminosidade e ângulo de tinta. E conforme Camargos; Gonçalez (2001) quando a madeira apresentar valor da luminosidade igual ou inferior a 56 será considerada escura.

Para os três grupos ocorreram diferenças estatísticas significativas para massa específica e parâmetros colorimétricos pelo teste de Scott-Knott (Tabelas 2 e 3). O mesmo ocorreu com o trabalho realizado por Silva et al. (2015), que estudaram oito espécies nativas do Rio Grande do Sul, que denotaram diferenças estatística significativa para as espécies.

A partir da análise estatística (Figura 2) observou-se que há diferença estatística significativa entre os planos tangencial e transversal para todos os parâmetros exceto para o ângulo de tinta (h), e o plano tangencial apresentou coloração mais clara que o transversal. Atayde et al. (2011) encontraram diferença estatística significativa para todos os parâmetros dos planos tangencial e transversal para a madeira Brosimum sp. Conforme estes autores, as condições dos sítios podem ter influenciado no desenvolvimento da planta, na qualidade da madeira e suas propriedades. E conforme Nishino (2000), no plano transversal as fibras podem reduzir a luminosidade, não em virtude dos orifícios dos lumens, mas por causa da luminosidade das substâncias na parede celular.

Figura 2. Teste de média dos parâmetros colorimétricas dos planos tangencial e transversal de espécies madeireiras do estado de Mato Grosso.

Figure 2. Mean test of the colorimetric parameters of the tangential and transversal surfaces of wood species in the state of Mato Grosso.

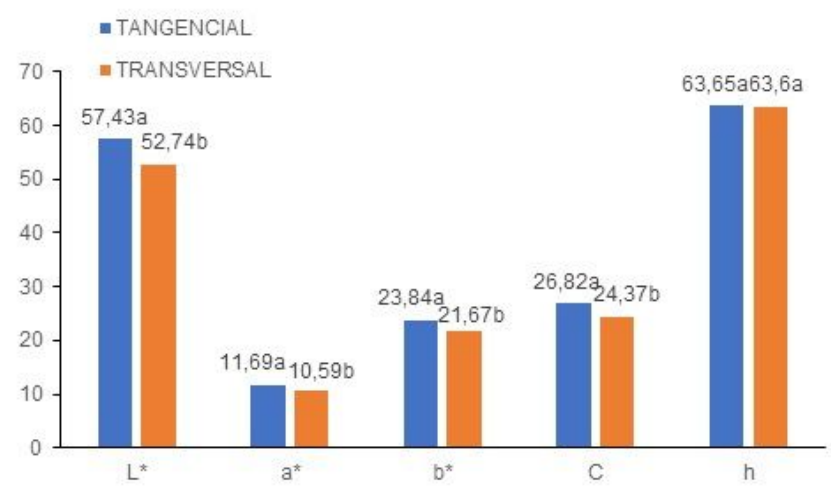


Médias seguidas de mesmas letras minúsculas na coluna, não diferem estatisticamente entre si pelo teste de Scott-Knott, a 5\% de probabilidade. Onde: L: luminosidade; $\mathrm{a}^{\star}$ e $\mathrm{b}^{\star}$ : coordenadas cromáticas dos eixos verde-vermelho e azulamarelo, respectivamente; C: saturação da cor; h: ângulo de tonalidade.

$\mathrm{Na}$ Tabela 5, encontra-se a matriz de correlação entre os parâmetros colorimétricos da face longitudinal tangencial.

Os parâmetros que tiveram maior correlação foram entre o eixo azul-amarelo $\left(b^{\star}\right)$ e a saturação (C), pois esse parâmetro depende das coordenadas cromáticas $\mathrm{a}^{\star} \mathrm{e} \mathrm{b}^{\star}$. As espécies Clarisia racemosa, Apuleia sp. e Parkia sp., por exemplo, obtiveram maiores valores de $\mathrm{b}^{\star}$ e altos valores para a saturação. Como as madeiras tiveram uma tendência de ter cores amareladas, o eixo verde-vermelho $\left(\mathrm{a}^{\star}\right)$ não teve tanta influência na saturação (C). Já o ângulo de tinta (h) teve uma correlação negativa com o eixo verde-vermelho $\left(a^{\star}\right)$.

Melo et al. (2013) também encontraram correlações positivas para a madeira de Schizolobium amazonicum entre o eixo azul-amarelo $\left(b^{*}\right)$ e a saturação $(C)$, e entre a luminosidade $\left(\mathrm{L}^{\star}\right)$ e o ângulo de tinta $(\mathrm{h})$, já para o eixo verdevermelho $\left(\mathrm{a}^{\star}\right)$ e ângulo de tinta (h) também obtiveram correlação negativa.
A massa específica apresentou correlação positiva com o eixo verde-vermelho $\left(\mathrm{a}^{\star}\right)$. Mori et al. (2004) afirmam que a medida que o teor de polifenóis aumenta na madeira, provavelmente a cor avermelhada também irá aumentar, e a presença de tais constituintes está relacionada ao acréscimo na massa específica da madeira (SILVA et al., 2015). Esses últimos autores também encontraram correlação positiva para o eixo-verde vermelho $\left(\mathrm{a}^{\star}\right)$ e massa específica.

Já com o a luminosidade $\left(\mathrm{L}^{*}\right)$ e ângulo de tinta $(\mathrm{h})$, a massa específica obteve correlações negativas, provavelmente isso ocorreu pelo fato das madeiras estudadas de cores amarelas e brancas tenderem a ter menores massas específicas, ou seja, quanto maior for o parâmetro de luminosidade $\left(\mathrm{L}^{\star}\right)$ e ângulo de tinta (h) menor será a massa específica, pois o ângulo de tinta $(\mathrm{h})$ estará mais próximo do eixo azul-amarelo $\left(\mathrm{b}^{\star}\right)$.. Silva et al. (2015) encontraram correlação negativa entre a massa específica e luminosidade na seção tangencial para as espécies Araucaria angustifolia (Bertol.) Kuntze, Myrocarpus frondosus Allemao e Luehea divaricata Mart. \& Zucc. Melo et al. (2013) e Mori et al. (2004) não encontraram correlação significativa entre a massa específica e os parâmetros colorimétricos.

Tabela 5. Correlação de Pearson para os parâmetros colorimétricos e massa específica da face tangencial das madeiras das principais espécies comercializadas no estado de Mato Grosso.

Table 5. Pearson correlation for the colorimetric parameters and density based on the tangential direction of the main woods commercialized in the state of Mato Grosso.

\begin{tabular}{c|c|c|c|c|c}
\hline $\begin{array}{c}\text { Parâmetros } \\
\text { colorimétricos }\end{array}$ & $\mathbf{a}^{*}$ & $\mathbf{b}^{*}$ & $\mathbf{C}$ & $\mathbf{h}$ & $\mathbf{d}$ \\
\hline $\mathrm{L}^{*}$ & $-0,52^{\star}(0,0031)$ & $0,612^{\star}(0,0003)$ & $0,40^{\star}(0,0297)$ & $0,75^{\star}(<, 0001)$ & $-0,71^{\star}(<, 0001)$ \\
$\mathrm{a}^{*}$ & & $0,025(0,8957)$ & $0,34(0,0652)$ & $-0,85^{\star}(<, 0001)$ & $0,39^{\star}(0,0339)$ \\
$\mathrm{b}^{*}$ & & $0,93^{\star}(<, 0001)$ & $0,52^{\star}(0,0031)$ & $-0,22(0,2425)$ \\
$\mathrm{C}$ & & & $0,18(0,3510)$ & $-0,07(0,7052)$ \\
$\mathrm{h}$ & & & & $-0,43^{\star}(0,0179)$ \\
\hline
\end{tabular}

Entre parênteses o p-valor ao nível de $5 \%$ de significância. Onde: L: luminosidade; $\mathrm{a}^{\star}$ e $\mathrm{b}^{\star}$ : coordenadas cromáticas dos eixos verde-vermelho e azul-amarelo, respectivamente; C: saturação da cor; h: ângulo de tonalidade; d: massa específica. ${ }^{\star}$ Valores com correlação significativa. 


\section{Conclusões}

As cores marrom-oliva, amarelo-amarronzado e marrom-claro foram as mais frequentes. As madeiras tiveram uma tendência de ser mais amarelas que vermelhas.

A seção transversal possui coloração mais escuras que a seção tangencial.

As madeiras mais claras apresentaram menores massas específicas, e as mais escuras resultaram em maiores massas específicas.

\section{Agradecimentos}

À CAPES pela concessão de bolsa de mestrado ao primeiro autor e ao INDEA-MT pelo apoio cedido.

\section{Referências}

AMORIM, P. G. R.; GONÇALEZ, J. C.; CAMARGOS, J. A. Propriedades da madeira de Pinus caribaea e Eucalyptus grandis estimadas por colorimetria. Cerne, Lavras, v. 19, n. 3, p. 461-466, 2013. http://dx.doi.org/10.1590/S010477602013000300013 .

ATAYDE, C. M.; GONÇALEZ, J. C.; CAMARGOS, J. A. Características colorimétricas entre as seções anatômicas da madeira de muirapiranga (Brosimum sp.). Cerne, Lavras, v. 17, n. 2, p. 231-235, 2011. http://dx.doi.org/10.1590/S010477602011000200011 .

BARROS, S. V. S.; MUNIZ, G. I. B.; MATOS, J. L. M. Caracterização colorimétrica das madeiras de três espécies da Amazônia. Cerne, Lavras, v. 20, n. 3, p. 337-342, 2014. http://dx.doi.org/10.1590/01047760201420031421.

CAMARGOS, J. A.; GONÇALEZ, J. C. A colorimetria aplicada como instrumento na elaboração de uma tabela de cores de madeira. Brasil Florestal, Brasília, n. 71, p. 30-41, 2001.

COSTA, J. A.; GONÇALEZ, J. C.; CAMARgOS, J. A. A.; GOMES, I. A. S. Fotodegradação de duas espécies de madeiras tropicais: jatobá (Hymenaea courbaril) e tauari (Couratari oblongifolia), submetidas à radiação ultravioleta. Cerne, Lavras, v. 17, n. 1, p. 133-139, 2011. http://dx.doi.org/10.1590/S0104-77602011000100016.
CRUZ, C.; REGAZZI, A. J. Modelos biométricos aplicados ao melhoramento genético. Viçosa: UFV, p. 390, 2001.

DIAS JÚNIOR, A. F.; CARVALHO, A. M.; SANTOS, P. V.; SILVA, M. A. Usinagem da madeira de cinco espécies nativas brasileiras. Revista Eletrônica em Gestão Educação e Tecnologia ambiental - REGET, Santa Maria, v. 18, n. 3, p. 1200-1206, 2014. http://dx.doi.org/10.5902/2236117014261.

INSTITUTO DE DEFESA AGROPECUÁRIA DO ESTADO DE MATO GROSSO - INDEA-MT. Noções básicas de anatomia e identificação macroscópica de madeiras. Cuiabá: INDEA, p. 12, 2011.

GARCIA, R. A.; OLIVEIRA, N. S.; NASCIMENTO, A. M.; SOUZA, N. D. Colorimetria de madeiras dos gêneros Eucalyptus e Corymbia e sua correlação com a densidade. Cerne, Lavras, v. 20, n. 4, p. 509-517, 2014. http://dx.doi.org/10.1590/01047760201420041316.

GREY, T. Color Confidence: The Digital Photographer's Guide to Color Management. Second Edition. Indianapolis: Wiley Publishing, p. 256, 2006.

GROBÉRIO, M. P.; LAHR, F. A. R. Indicações para o emprego da madeira de espécies tropicais do Brasil. Madeira: arquitetura e engenharia, v. 3, n. 8, p. 11, 2002.

LAVISCI, P.; JANIN, G.; UZIELLI, L. Qualité du bois de six essences du maquis méditerranéen. Forêt Méditerranéenne, v. 11, n. 1, p. 69-78, 1989.

LEÃO, A. C.; ARAúJO, A. A.; SOUZA, L. A. C. Implementação de sistema de gerenciamento de cores para imagens digitais. PUC-Minas: Poços de Caldas, p.38, 2005.

MANLY, B. J. F. Métodos estatísticos multivariados: uma introdução. Porto Alegre: Bookman, p. 229, 2008.

MELO, R. R.; DEL MENEZZI, C. H. S.; SOUZA, M. R.; STANGERLIN, D. M. Avaliação das propriedades físicas, químicas, mecânicas e de superfície de lâminas de paricá (Schizolobium amazonicum Huber ex. Ducke). Floresta e Ambiente, Seropédica, v. 20, n. 2, p. 238-249, 2013. http://dx.doi.org/10.4322/floram.2013.004.

MORI, C. L. S. O.; LIMA, J. T.; MORI, F. A.; TRUGILHO, P. F.; GONÇALEZ, J. C. Caracterização da cor da madeira de clones de híbridos de Eucalyptus spp. Cerne, Lavras, v. 11, n. 2, p. 137-146, 2005. 
MORI, C. L. S. O.; MORI, F. A.; LIMA, J. T.; TRUGILHO, P. F.; OLIVEIRA, A. C. Influência das características tecnológicas na cor da madeira de eucaliptos. Ciência Florestal, Santa Maria, v.14, n.2, p.123-132, 2004. http://dx.doi.org/10.5902/198050981812.

NISHINO, Y.; JANIN, G.; CHANSON, B.; DÉTIENNE, P.; GRIL, J.; THIBAUT, B. Colorimetry of wood specimens from French Guiana. Wood Science, v. 44, p. 3-8, 1998. https://doi.org/10.1007/BF00521867.

NISHINO, Y.; JANIN, G.; YAMADA, Y.; KITANO, D. Relations between the colorimetric values and densities of sapwood. Wood Science, v.46, p. 267-272, 2000. https://doi.org/10.1007/BF00766215.

PAULA, M. H.; MESQUITA, R. R. S.; GONÇALEZ, J. C.; RIBEIRO, E. S.; SOUZA, R. S. Utilização de métodos não destrutivos para caracterização simplificada da madeira de cumaru (Dipteryx odorata Willd). Biodiversidade, Rondonópolis, v. 15, n. 2, p. 136-149, 2016.

PERTUZZATTI, A.; MISSIO, A. L.; CONTE, B.; SOUZA, S. C.; SANTINI, E. J.; HASELEIN, C. R. Propriedades físicas da madeira de Pinus elliotti var. elliottii termorretificada sob diferentes atmosferas. Ciência da Madeira, Pelotas, v. 7, n. 1, p. 7-15, 2016. http://dx.doi.org/10.12953/21776830/rcm.v7n1p7-15.

PINCELLI, A. L. P. S. M.; MOURA, L. F.; BRITO, J. O. Effect of thermal rectification on colors of Eucalyptus saligna and Pinus caribaea woods. Maderas, Ciencia y tecnologia, v. 14, n. 2, p. 239-248, 2012. http://dx.doi.org/10.4067/S0718221X2012000200010.

RAPPOLD, P. M.; SMITH, W. B. An investigation of the relantionships between harvest season, log, age, and drying schedule on the coloration of hard maple lumber. Forest Products Journal, v. 54, n. 12, p. 178-184, 2004.

ROMANINI, A.; STANGERLIN, D. M.; PARIZ, E.; SOUZA, A. P.; GATTO, D. A.; CALEGARI, L. Durabilidade natural da madeira de quatro espécies amazônicas em ensaios de deterioração de campo. Nativa, Sinop, v. 2, n. 1, p. 13-17, 2014. http://dx.doi.org/10.14583/2318-7670.v02n01a03 SECRETARIA DE ESTADO DO MEIO AMBIENTE DE MATO GROSSO - SEMA-MT. Beneficiamento e Comércio de Produtos da Madeira por Espécie Florestal. 2016.

SILVA, J. J. N.; CARDOSO, G. V.; SILVA JUNIOR, F. G.; STANGERLIN, D. M. Caracterização tecnológica da madeira de Schizolobium amazonicum para a produção de celulose
Kraft. Ciência da Madeira, Pelotas, v. 4, n. 1, p. 33-45, 2013. http://dx.doi.org/10.15210/cmad.v4i1.4049.

SILVA, E. S.; STANGERLIN, D. M.; GATTO, D. A.; CALEGARI, L.; PARIZ, E. Colorimetria da madeira de oito espécies nativas do estado do Rio Grande do Sul, Brasil. Ciência da Madeira, Pelotas, v. 6 n. 1, p. 31-37, 2015. http://dx.doi.org/10.15210/cmad.v6i1.4292.

SILVA, R. A. F.; SETTER, C.; MAZETTE, S. S.; MELO, R. R.; STANGERLIN, D. M. Colorimetria da madeira de trinta espécies tropicais. Ciência da Madeira, Pelotas, v. 8, n. 1, p. 36-41, 2017. http://dx.doi.org/10.15210/cmad.v8i1.9686.

STANGERLIN, D. M.; COSTA, A. F.; GARLET, A.; PASTORE, T. C. M. Resistência natural da madeira de três espécies amazônicas submetidas ao ataque de fungos apodrecedores. Ciência da Madeira, Pelotas, v. 4, n. 1, p. 1532, 2013. http://dx.doi.org/10.15210/cmad.v4i1.4056.

ZANUNCIO, A. J. V.; FARIAS, E. S.; SILVEIRA, T. A. Termorretificação e colorimetria da madeira de Eucalyptus grandis. Floresta e Ambiente, Seropédica v. 21 n. 1, p. 85-90, 2014. http://dx.doi.org/10.4322/floram.2014.005. 